\title{
Uniform and pointwise Bernstein-Walsh-type inequalities on a quasidisk in the complex plane
}

\author{
F.G. Abdullayev $\quad$ P. Özkartepe
}

\begin{abstract}
In this present work, we continue studying the Bernstein-Walsh type estimations for complex algebraic polynomials in the bounded and unbounded regions with quasiconformal boundary.
\end{abstract}

\section{Introduction and Definitions}

In many areas of research in mathematics (in approximation theory, embedding theory and etc.), one can be faced with two important problems given in the following: a) Determining how to undergo a change of (semi)norm of the holomorphic function when the given region expands; b) Determining the relationships between different (semi)norms of analytic functions in a given finite Jordan region on the complex plane in the various (semi)normed space

We will consider this problems for algebraic polynomials of complex variables in the well known Bergman space, and investigate the following problems: evaluating the increase of the modulus of polynomials in the exterior of the given region with respect to the norm of the polynomial in the this region; determining a change of (semi)norm of polynomials for the given region and, finally, combining obtained estimations for the modulus of polynomials, we will get the estimation modulus of polynomials in whole complex plane.

The first classical results of the first type belongs to Bernstein [13], Faber [15] and Walsh [28]. We will give this result in the right to our future arguments.

Received by the editors in April 2014 - In revised form in January 2016.

Communicated by H. De Schepper.

2010 Mathematics Subject Classification : Prim.30A10, 30C10, Sec.41A17.

Key words and phrases: Polynomials, Bernstein-Walsh inequalities, Conformal mapping, Quasiconformal curve. 
Let $G \subset \mathbb{C}$ be a bounded region with $0 \in G$ and the boundary $L:=\partial G$ being a closed Jordan curve, $\Omega:=\overline{\mathbb{C}} \backslash \bar{G}$, where $\overline{\mathbb{C}}:=\mathbb{C} \cup\{\infty\} ; \Delta:=\{w:|w|>1\}$. Let $w=\Phi(z)$ be the univalent conformal mapping of $\Omega$ onto $\Delta$ such that $\Phi(\infty)=$ $\infty, \lim _{z \rightarrow \infty} \frac{\Phi(z)}{z}>0$ and for $R>1$, we've $L_{R}:=\{z:|\Phi(z)|=R\}, G_{R}:=$ int $L_{R}$, $\Omega_{R}:=\operatorname{ext}_{R}$.

Let $\wp_{n}$ denote the class of all complex algebraic polynomials $P_{n}(z)$ of degree at most $n, n \in \mathbb{N}:=\{1,2, \ldots\}$. According to Bernstein-Walsh lemma [28], for any $z \in \Omega$ we have

$$
\left\|P_{n}\right\|_{C\left(\bar{G}_{R}\right)} \leq|\Phi(z)|^{n}\left\|P_{n}\right\|_{C(\bar{G})},
$$

where

$$
\left\|P_{n}\right\|_{C(\bar{G})}:=\max _{z \in \bar{G}}\left|P_{n}(z)\right|,
$$

and consequently, for $z \in \bar{G}_{R}$ :

$$
\left\|P_{n}\right\|_{C\left(\bar{G}_{R}\right)} \leq R^{n}\left\|P_{n}\right\|_{C(\bar{G})} .
$$

By taking $R=1+\frac{\text { const. }}{n}$, we fund that, the norm $\left\|P_{n}\right\|_{C(\bar{G})}$ increases no more than a constant, when the domain $G$ is expanded to $G_{R}$.

Let $h(z)$ be the weight function, which is defined in $G_{R}$, for some $R>1$. For any $p>0$ we introduce:

$$
\begin{aligned}
\left\|P_{n}\right\|_{A_{p}(h, G)}: & =\left(\iint_{G} h(z)\left|P_{n}(z)\right|^{p} d \sigma_{z}\right)^{1 / p}<\infty, \\
\left\|P_{n}\right\|_{A_{p}(G)}: & =\left\|P_{n}\right\|_{A_{p}(1, G)},
\end{aligned}
$$

where $\sigma_{z}$ is the two-dimensional Lebesgue measure and

$$
\begin{aligned}
\left\|P_{n}\right\|_{\mathfrak{L}_{p}(h, L)}: & :=\left(\int_{L} h(z)\left|P_{n}(z)\right|^{p}|d z|\right)^{1 / p}<\infty, \\
\left\|P_{n}\right\|_{\mathfrak{L}_{p}(L)}: & :=\left\|P_{n}\right\|_{\mathfrak{L}_{p}(1, L)},
\end{aligned}
$$

if $L$ is rectifiable. The same effect, analogously to (1.3), is observed for the $\left\|P_{n}\right\|_{\mathfrak{L}_{p}(L)}$ according to the following estimate [16]:

$$
\left\|P_{n}\right\|_{\mathfrak{L}_{p}\left(L_{R}\right)} \leq R^{n+\frac{1}{p}}\left\|P_{n}\right\|_{\mathfrak{L}_{p}(L)}, p>0
$$

Let the function $\varphi$ maps $G$ conformally and univalently onto $B:=\{w$ : $|w|<1\}$ which is normalized by $\varphi(0)=0, \varphi^{\prime}(0)>0$, and $\psi:=\varphi^{-1}$.

Definition 1. [22, p.286]A bounded Jordan region $G$ is called a $k$-quasidisk, $0 \leq k<$ 1 , if any conformal mapping $\psi$ can be extended to a $K$-quasiconformal, $K=\frac{1+k}{1-k}$, homeomorphism of the plane $\overline{\mathbb{C}}$ on the $\overline{\mathbb{C}}$. In that case the curve $L:=\partial G$ is called $a$ $k$-quasicircle. The region $G$ (curve $L$ ) is called a quasidisk (quasicircle), if it is $k$-quasidisk ( $k$-quasicircle) with some $0 \leq k<1$. 
It is well known that quasicircle may not even be locally rectifiable [17, p.104].

The Bernstein-Walsh type estimation in the $A_{p}(h, G)$ for quasidisks and for some weight function $h(z)$, was proved in [3] (see: Part 2, Lemma 23). In particular, for $h(z) \equiv 1$,

$$
\left\|P_{n}\right\|_{A_{p}\left(G_{R}\right)} \leq c_{1}\left[1+c_{2}(R-1)\right]^{n+\frac{1}{p}}\left\|P_{n}\right\|_{A_{p}(G)}, p>0,
$$

where $c_{1}=c_{1}\left(G, p, c_{2}\right)>0$ and $c_{2}(G, p)>0$ are constants independent of $R$ and $n$.

So, taking $R=1+\frac{\text { const. }}{n}$, from (1.7) we see that $\left\|P_{n}\right\|_{A_{p}\left(G_{R}\right)}$ and $\left\|P_{n}\right\|_{A_{p}(G)}$ are equivalent.

N. Stylianopoulos [24] replaced the norm $\left\|P_{n}\right\|_{C(\bar{G})}$ with norm $\left\|P_{n}\right\|_{A_{2}(G)}$ on the right-hand side of (1.1) and, introduced a new version of the Bernstein-Walsh lemma as following the type: Assume that $L$ is quasicircle and rectifiable. Then there exists a constant $c=c(L)=$ const. $>0$ such that, for any $n \in \mathbb{N}$ and $P_{n} \in \wp_{n}$

$$
\left|P_{n}(z)\right| \leq c \frac{\sqrt{n}}{d(z, L)}\left\|P_{n}\right\|_{A_{2}(G)}|\Phi(z)|^{n+1}, z \in \Omega,
$$

where $d(z, L):=\operatorname{dist}(z, L):=\inf \{|z-\zeta|: \zeta \in L\}$.

Second problem which we will investigate in this work, is the following:

$$
\left\|P_{n}\right\|_{C(\bar{G})} \leq v_{n}\left\|P_{n}\right\|_{A_{p}(G)}
$$

where $v_{n}:=v_{n}(G, p) \rightarrow \infty, n \rightarrow \infty$, depending on the geometrical properties of the region $G$. Inequalities analogously to (1.9) in the literature are often found under the "Nikol'skii-type inequality".

Examples of inequalities type of (1.8) and (1.9) for the polynomials we can indicate the investigations by Nikol'skii [21], Mamedhanov [19], Milovanovic et al.[20], Abdullayev et al. [3]-[7], Andrievskii [11], Stylianopoulos [24], [25] and others.

\section{Main Results}

Throughout this paper, we will denote by $c, c_{0}, c_{1}, c_{2}, \ldots$ which are positive constants and by $\varepsilon_{0}, \varepsilon_{1}, \varepsilon_{2}, \ldots$ sufficiently which are small positive constants (in general, different in different relations) that depends on $G$ in general and on parameters inessential for the argument; otherwise, such dependence will be explicitly stated.

First of all we note that, for any $p>0$, according to mean value theorem, there exists a constant $c_{4}=c_{4}(G)>0$, independent from $n$ and $z$, such that for any Jordan region $G$ and $P_{n} \in \wp_{n}$, the following is true:

$$
\left|P_{n}(z)\right| \leq c_{4}\left(\frac{1}{d(z, L)}\right)^{\frac{2}{p}}\left\|P_{n}\right\|_{A_{p}(G)}, z \in G .
$$


Combining (1.8) and (2.1), we obtain an estimation on the growth of $\left|P_{n}(z)\right|$ in $G$ and $\Omega$ as the following: if $L$ is quasicircle and rectifiable, then for any $P_{n} \in \wp_{n}, n \in$ $\mathbb{N}$,

$$
\left|P_{n}(z)\right| \leq \frac{c_{5}}{d(z, L)}\left\|P_{n}\right\|_{A_{2}(G)}\left\{\begin{array}{cc}
1, & z \in G \\
\sqrt{n}|\Phi(z)|^{n+1}, & z \in \Omega .
\end{array}\right.
$$

It shows that, if we know the behavior of the $\left|P_{n}(z)\right|$ for $z \in L=\partial G$, then we can find the estimation for the $\left|P_{n}(z)\right|$ in the whole complex plane. In particular, according to [5], we have the following:

Theorem A. Assume that $G$ is a $k$-quasidisk. Then there exists a constant $c_{6}=c_{6}(G, p)>0$ such that, for any $n \in \mathbb{N}$ and $P_{n} \in \wp_{n}$ the following is true:

$$
\left|P_{n}(z)\right| \leq c_{6} n^{\frac{2(1+k)}{p}}\left\|P_{n}\right\|_{A_{p}(G)}, \quad z \in \bar{G} .
$$

Therefore, combining the estimation (2.3) with (1.8), we obtain an estimation on the growth of $\left|P_{n}(z)\right|$ in the whole complex plane:

Corollary B. Let $L$ be a $k$-quasicircle for some $0 \leq k<1$ and rectifiable. Then for any $n \in \mathbb{N}$ and $P_{n} \in \wp_{n}$

$$
\left|P_{n}(z)\right| \leq c_{6}^{\prime}\left\|P_{n}\right\|_{A_{2}(G)}\left\{\begin{array}{cc}
n^{1+k}, & z \in \bar{G} \\
\frac{\sqrt{n}}{d(z, L)}|\Phi(z)|^{n+1}, & z \in \Omega
\end{array}\right.
$$

where $c_{6}^{\prime}=\max \left\{c, c_{6}\right\}$.

In this work, we study a similar problem to (1.8), (2.2) and (2.4) with respect to $\left\|P_{n}\right\|_{A_{p}(G)}, p>0$, for regions with quasiconformal boundary (not necessarily rectifiable!).

Now, we start to formulate the new results.

Theorem 2. Assume that $G$ is quasidisk; $P_{n} \in \wp_{n}, n \in \mathbb{N}$, and $R_{1}=1+\frac{\varepsilon_{1}}{n}$. Then, for any $p>0$

$$
\left|P_{n}(z)\right| \leq c_{7}\left(\frac{\sqrt{n}}{d\left(z, L_{R_{1}}\right)}\right)^{\frac{2}{p}}\left\|P_{n}\right\|_{A_{p}(G)}|\Phi(z)|^{n+1}, \quad z \in \Omega_{R_{1}},
$$

where $c_{7}=c_{7}(G, p)>0$.

If we use a similar estimation to (2.1), but rewriting for points $z \in G_{R}$, then according (2.5), we obtain:

Corollary 3. Let $G$ be a quasidisk; $P_{n} \in \wp_{n}, n \in \mathbb{N}$, and $R_{1}=1+\frac{\varepsilon_{1}}{n}$. Then for any $p>0$

$$
\left|P_{n}(z)\right| \leq c_{8}\left(\frac{1}{d\left(z, L_{R_{1}}\right)}\right)^{\frac{2}{p}}\left\|P_{n}\right\|_{A_{p}(G)}\left\{\begin{array}{cl}
1, & z \in G_{R_{1}}, \\
\sqrt{n}|\Phi(z)|^{n+1}, & z \in \Omega_{R_{1}},
\end{array}\right.
$$

where $c_{8}=c_{8}(G, p)>0$.

Now, combining (2.3) and Theorem 2, by (1.3) we have: 
Corollary 4. Let $G$ be a $k$-quasidisk for some $0 \leq k<1 ; P_{n} \in \wp_{n}, n \in \mathbb{N}$, and $R_{1}:=1+\frac{\varepsilon_{1}}{n}$. Then, for $p>0$

$$
\left|P_{n}(z)\right| \leq c_{9}\left\|P_{n}\right\|_{A_{p}(G)}\left\{\begin{array}{cl}
n^{\frac{2(1+k)}{p},} & z \in \bar{G}_{R_{1}}, \\
\left(\frac{\sqrt{n}}{d\left(z, L_{R_{1}}\right)}\right)^{\frac{2}{p}}|\Phi(z)|^{n+1}, & z \in \Omega_{R_{1}},
\end{array}\right.
$$

where $c_{9}=c_{9}(G, p)>0$.

Thus, Corollaries 3 and 4 are provide an opportunity to observe the growth of $\left|P_{n}(z)\right|$ on the whole complex plane. From the conditions of the theorem we see that it holds for $k$-quasidisks with $0 \leq k<1$. But as we know, calculating the coefficient of quasiconformality for some curves is not an easy task. Therefore, we define a more general class of regions with another characteristics. One of them is the following definition.

Definition 5. [4]We say that $G \in Q_{\alpha}, 0<\alpha \leq 1$, if

i) $L=\partial G$ is quasicircle,

ii) $\Phi \in H^{\alpha}(\bar{\Omega})$ for some $0<\alpha \leq 1$.

For $G \in Q_{\alpha}$ we have the following result.

Theorem 6. Assume that $G \in Q_{\alpha}$ for some $\frac{1}{2} \leq \alpha \leq 1 ; P_{n} \in \wp_{n}, n \in \mathbb{N}$, and $R_{1}:=1+\frac{\varepsilon_{1}}{n}$. Then, for any $p \geq 1$

$$
\left|P_{n}(z)\right| \leq c_{10}\left(\frac{n^{\frac{1}{\alpha}}}{d\left(z, L_{R_{1}}\right)}\right)^{\frac{1}{p}}\left\|P_{n}\right\|_{A_{p}(G)}|\Phi(z)|^{n+1}, z \in \Omega_{R_{1}},
$$

where $c_{10}=c_{10}(G, p)>0$.

Analogous to (2.3), for $G \in Q_{\alpha}, \frac{1}{2} \leq \alpha \leq 1$, we can find (see, for example, [5, Th.5.2]) a constant $c_{11}=c_{11}(G, \alpha, p)>0$ such that for any $P_{n} \in \wp_{n}, n \in \mathbb{N}$ and $p>1$, the following is true:

$$
\left|P_{n}(z)\right| \leq c_{11} n^{\frac{2}{p \alpha}}\left\|P_{n}\right\|_{A_{p}(G)}, z \in \bar{G}
$$

In this case, combining Theorem 6 and (2.9), we obtain an estimate for $\left|P_{n}(z)\right|$ on the whole complex plane.

Corollary 7. Let $G \in Q_{\alpha}$ for some $\frac{1}{2} \leq \alpha \leq 1 ; P_{n} \in \wp_{n}, n \in \mathbb{N}$, and $R_{1}:=1+\frac{\varepsilon_{1}}{n}$. Then, for any $p>1$

$$
\left|P_{n}(z)\right| \leq c_{12}\left\|P_{n}\right\|_{A_{p}(G)}\left\{\begin{array}{cl}
n^{\frac{2}{p \alpha}}, & z \in \bar{G}_{R_{1}}, \\
\left(\frac{n^{\frac{1}{\alpha}}}{d\left(z, L_{R_{1}}\right)}\right)^{\frac{1}{p}}|\Phi(z)|^{n+1}, & z \in \Omega_{R_{1}}
\end{array}\right.
$$

where $c_{12}=c_{12}(G, \alpha, p)>0$. 
Theorem 6 holds for all $p \geq 1$. If we exclude the case $p=1$, then the right hand side of (2.8) can be improved for $\frac{1}{2} \leq \alpha \leq 1$ and $p>1$. For this reason, we give the following definition.

Definition 8. We say that $G \in Q_{\alpha}^{\beta}$, if $G \in Q_{\alpha}$ for some $0<\alpha \leq 1$ and $\Psi \in H^{\beta}(|w| \geq$ 1) for some $0<\beta \leq 1$.

For $G \in Q_{\alpha}^{\beta}$ we get :

Theorem 9. Assume that $G \in Q_{\alpha}^{\beta}$ for some $\frac{1}{2} \leq \alpha \leq 1$ and $0<\beta \leq 1 ; P_{n} \in \wp_{n}$, $n \in \mathbb{N}$, and $R_{1}:=1+\frac{\varepsilon_{1}}{n}$. Then, for any $p>1$

$$
\left|P_{n}(z)\right| \leq \frac{c_{13}\left\|P_{n}\right\|_{A_{p}(G)}|\Phi(z)|^{n+1}}{d\left(z, L_{R_{1}}\right)}\left\{\begin{array}{ll}
n^{\frac{1}{\alpha}\left(\frac{2}{p}-1\right)}, & 2-\alpha<p<2, \\
\sqrt{n}, & p=2, \\
n^{(1-\beta)\left(1-\frac{2}{p}\right)}, & p>2,
\end{array} \quad z \in \Omega_{R_{1}}\right.
$$

where $c_{13}=c_{13}(G, \alpha, \beta, p)>0$.

Clearly, we see that the estimate (2.11) is better than (2.8) for any $2-\alpha<p \leq$ $2+\frac{1}{\alpha \beta}$.

Remark 10. We note, that the class $Q_{\alpha}^{\beta}$ is sufficiently large. For example,

a) If $L=: \partial G$ is a smooth curve having continuous tangent line, then $G \in Q_{\alpha}^{\beta}$ for all $0<\alpha, \beta<1$.

b) If $G$ is "L-shaped" region, then $\Phi \in \operatorname{Lip} \frac{2}{3}$ and $\Psi \in \operatorname{Lip} \frac{1}{2}$.

c) If $L$ is quasismooth (in the sense of Lavrentiev), that is, for every pair $z_{1}, z_{2} \in L$, if $s\left(z_{1}, z_{2}\right)$ represents the smallest of the lengths of the arcs joining $z_{1}$ to $z_{2}$ on $L$, there exists a constant $c>1$ such that $s\left(z_{1}, z_{2}\right) \leq c\left|z_{1}-z_{2}\right|$, then $\Phi \in$ Lip $\alpha$ for $\alpha=\frac{1}{2}\left(1-\frac{1}{\pi} \arcsin \frac{1}{c}\right)^{-1}$ and $\Psi \in \operatorname{Lip} \beta$ for $\beta=\frac{2}{(1+c)^{2}}$ [26], [27].

d) If $L$ is "c-quasiconformal" (see, for example, [18]), then $\Phi \in \operatorname{Lip} \alpha$ for $\alpha=\frac{\pi}{2\left(\pi-\arcsin \frac{1}{c}\right)}$ and $\Psi \in \operatorname{Lip} \beta$ for $\beta=\frac{2\left(\arcsin \frac{1}{c}\right)^{2}}{\pi\left(\pi-\arcsin \frac{1}{c}\right)}$. Also, if $L$ is an asymptotic conformal curve, then $\Phi \in \operatorname{Lip} \alpha$ and $\Psi \in \operatorname{Lip} \beta$ for all $0<\alpha, \beta<1$ [18].

Therefore, we can calculate $\alpha$ and $\beta$ in the right parts of estimates (2.8)-(2.11) for each case, respectively.

In case $p=2$, for the quasidisks, we have the following general result.

Theorem 11. Assume that $G$ is quasidisk; $P_{n} \in \wp_{n}, n \in \mathbb{N}$. Then, there exists a constant $c_{14}=c_{14}(G)>0$ such that for any $R>1$ we have

$$
\left|P_{n}(z)\right| \leq c_{14} \frac{\sqrt{n}}{d\left(z, L_{R}\right)}\left\|P_{n}\right\|_{A_{2}\left(G_{R}\right)}|\Phi(z)|^{n+1}, z \in \Omega_{R}
$$

Now, combining estimation (1.3) and (2.3) with Theorem 11, we also get a result in Corollary 4.

Thus, to estimation the quantity $\left|P_{n}(z)\right|$ in $\bar{G}_{1+\frac{c}{n}}$, we need to know a similar estimate for $\left|P_{n}(z)\right|$ in $\bar{G}$, and by using (1.3), we get the desired estimation. Therefore, (2.12) is meaningful for $z \in \Omega$ such that "not too close" to the boundary. In this regard, we give the following result. 
Corollary 12. For any compact subset $F \Subset \Omega$ and $P_{n} \in \wp_{n}, n \in \mathbb{N}$, we have

$$
\left|P_{n}(z)\right| \leq c_{15} \frac{\sqrt{n}}{d(z, L)}\left\|P_{n}\right\|_{A_{2}(G)}|\Phi(z)|^{n+1}, z \in F \Subset \Omega,
$$

where $c_{15}=c_{15}(G, F)>0$.

Theorems 2, 6, 9 and their corollaries can be formulated for the point $z \in \Omega$. For this reasion we need the following notation.

Let $L$ be a $K$-quasiconformal and $y($.$) be a regular quasiconformal reflection$ across $L$ (for details see Part 2). For any $R>1$, we put $L^{*}:=y\left(L_{R}\right), G^{*}:=$ int $L^{*}, \Omega^{*}:=\operatorname{ext} L^{*}$; denote by $\Phi_{R}$ the conformal mapping of $\Omega^{*}$ onto $\Delta$ with the normalization $\Phi_{R}(\infty)=\infty, \lim _{z \rightarrow \infty} \frac{\Phi_{R}(z)}{z}>0$, and let $\Psi_{R}:=\Phi_{R}^{-1}$.

Now, we assume that the point $z \in \Omega$ is arbitrarily close to the bound of $L$. In this case, we will replace the function $\Phi(z)$ with $\Phi_{R}(z)$, which is shown in the following theorem.

Theorem 13. Assume that $G$ is a quasidisk; $R=1+\frac{1}{n}$ and $P_{n} \in \wp_{n}, n \in \mathbb{N}$. Then, for any $p>0$

$$
\left|P_{n}(z)\right| \leq c_{16}\left(\frac{\sqrt{n}}{d(z, L)}\right)^{\frac{2}{p}}\left\|P_{n}\right\|_{A_{p}(G)}\left|\Phi_{R}(z)\right|^{n+1}, \quad z \in \Omega,
$$

where $c_{16}=c_{16}(G, p)>0$.

Using (2.3), analogously to Corollary 4, we have:

Corollary 14. Let $G$ be a $k$-quasidisk for some $0 \leq k<1$, then for any $P_{n} \in \wp_{n}$, $n \in \mathbb{N}$,

$$
\left|P_{n}(z)\right| \leq c_{17}\left\|P_{n}\right\|_{A_{2}(G)}\left\{\begin{array}{cc}
n^{(1+k)}, & z \in \bar{G} \\
\frac{\sqrt{n}}{d(z, L)}\left|\Phi_{R}(z)\right|^{n+1}, & z \in \Omega,
\end{array}\right.
$$

where $c_{17}=c_{17}(G)>0$.

Analogous, for the regions $G \in Q_{\alpha}$ and $G \in Q_{\alpha}^{\beta}$ we get:

Theorem 15. Assume that $G \in Q_{\alpha}$ for $\frac{1}{2} \leq \alpha \leq 1 ; R=1+\frac{1}{n}$ and $P_{n} \in \wp_{n}, n \in \mathbb{N}$. Then, for any $p \geq 1$

$$
\left|P_{n}(z)\right| \leq c_{18}\left(\frac{n^{\frac{1}{\alpha}}}{d(z, L)}\right)^{\frac{1}{p}}\left\|P_{n}\right\|_{A_{p}(G)}\left|\Phi_{R}(z)\right|^{n+1}, z \in \Omega,
$$

where $c_{18}=c_{18}(G, \alpha, p)>0$.

Theorem 16. Assume that $G \in Q_{\alpha}^{\beta}$ for some $\frac{1}{2} \leq \alpha \leq 1$ and $0<\beta \leq 1 ; R=1+\frac{1}{n}$, and $P_{n} \in \wp_{n}, n \in \mathbb{N}$. Then, for any $p>1$

$$
\left|P_{n}(z)\right| \leq \frac{c_{19}\left\|P_{n}\right\|_{A_{p}(G)}\left|\Phi_{R}(z)\right|^{n+1}}{d(z, L)}\left\{\begin{array}{ll}
n^{\frac{1}{\alpha}\left(\frac{2}{p}-1\right)}, & 2-\alpha<p<2, \\
\sqrt{n}, & p=2, \\
n^{(1-\beta)\left(1-\frac{2}{p}\right)}, & p>2,
\end{array} \quad z \in \Omega,\right.
$$

where $c_{19}=c_{19}(G, \alpha, \beta, p)>0$. 


\subsection{Sharpness of estimates}

The sharpness of the estimations (2.3)-(2.17) can be discussed by comparing them with the following result.

Theorem 17. a) For any $n \in \mathbb{N}$ there exist a polynomial $Q_{n}^{*} \in \wp_{n}$, region $G_{1}^{*} \subset \mathbb{C}$ and constant $c_{20}=c_{20}\left(G_{1}^{*}\right)>0$ such that for all $z \in \bar{G}_{1}^{*}$

$$
\left|Q_{n}^{*}(z)\right| \geq c_{20} n\left\|Q_{n}^{*}\right\|_{A_{2}\left(G_{1}^{*}\right)} ;
$$

b) For any $n \in \mathbb{N}$ there exist a polynomial $P_{n}^{*} \in \wp_{n}$, region $G_{2}^{*} \subset \mathbb{C}$ compact $F^{*} \Subset \Omega \backslash \bar{G}_{2}^{*}$ and constant $c_{21}=c_{21}\left(G_{2}^{*}, F^{*}\right)>0$ such that

$$
\left|P_{n}^{*}(z)\right| \geq c_{21} \frac{\sqrt{n}}{d(z, L)}\left\|P_{n}^{*}\right\|_{A_{2}\left(G_{2}^{*}\right)}|\Phi(z)|^{n+1}, z \in F \Subset \Omega \backslash \bar{G}_{2}^{*} .
$$

\section{Some auxiliary results}

The following definitions of quasiconformal curves are well known, see, for example, [8], [17, p.97] and [23]:

Definition 18. The Jordan arc (or curve) $L$ is called $K$-quasiconformal $(K \geq 1)$, if there is a $K$-quasiconformal mapping $f$ of the region $D \supset L$ such that $f(L)$ is a line segment (or circle).

We denote by $F(L)$ the set of all sense preserving plane homeomorphisms $f$ of the region $D \supset L$ such that $f(L)$ is a line segment (or circle) and let

$$
K_{L}:=\inf \{K(f): f \in F(L)\},
$$

where $K(f)$ is the maximal dilatation of a such mapping $f L$ is a quasiconformal curve, if $K_{L}<\infty$, and $L$ is a $K$-quasiconformal curve, if $K_{L} \leq K$.

Remark 19. It is well known that when we are not interested in the coefficients of quasiconformality of the curve L, then the definition of "quasicircle" and "quasiconformal curve" are equivalent. When we are interested with coefficients of quasiconformality of the given curve, then we will consider that if the curve $L$ is $K$-quasiconformal, then $L$ is $k$-quasicircle with $k=\frac{K^{2}-1}{K^{2}+1}$.

According to Remark 19, we will use both terms, depending on the situation.

Throughout this paper, for $a>0$ and $b>0$, we use the expression " $a \preceq b$ " (order inequality), if $a \leq c b$. The expression " $a \asymp b$ " means that " $a \preceq b$ " and " $b \preceq a$ " simultaneously.

We give some facts from the theory of quasi-conformal mapping, which will be used throughout of all proof below.

Let $L$ be a $K$-quasiconformal curve, then [8], there exists a quasiconformal reflection $y($.$) across L$ such that $y(G)=\Omega, y(\Omega)=G$ and $y($.$) is fixed the points$ of $L$. The quasiconformal reflection of $y($.$) satisfies the following condition [8],$ $[10$, p.26]: 


$$
\begin{aligned}
|y(\zeta)-z| & \asymp|\zeta-z|, z \in L, \varepsilon<|\zeta|<\frac{1}{\varepsilon} \\
\left|y_{\bar{\zeta}}\right| & \asymp\left|y_{\zeta}\right| \asymp 1, \varepsilon<|\zeta|<\frac{1}{\varepsilon} \\
\left|y_{\bar{\zeta}}\right| & \asymp|y(\zeta)|^{2},|\zeta|<\varepsilon,\left|y_{\bar{\zeta}}\right| \asymp|\zeta|^{-2},|\zeta|>\frac{1}{\varepsilon}
\end{aligned}
$$

and for the Jacobian $J_{y}=\left|y_{z}\right|^{2}-\left|y_{\bar{z}}\right|^{2}$ of $y($.$) the relation \left|y_{\bar{\zeta}}\right|^{2} \leq \frac{1}{1-k^{2}} J_{y}$ is hold, where $k=\frac{K^{2}-1}{K^{2}+1}$.

For any $R>1$ and $t>1$, we set $L^{*}:=y\left(L_{R}\right), L_{t}^{*}:=\left\{z:\left|\Phi_{R}(z)\right|=t\right\}$, $G_{t}^{*}:=\operatorname{int} L_{t}^{*}, \Omega_{t}^{*}:=\operatorname{ext} L_{t}^{*}$. According to [9], for all $z \in L^{*}$ and $t \in L$ such that $|z-t|=d(z, L)$, we have

$$
\begin{aligned}
d(z, L) & \asymp d\left(t, L_{R}\right) \asymp d\left(z, L_{R}^{*}\right) . \\
\left|\Phi_{R}(z)\right| & \leq\left|\Phi_{R}(t)\right| \leq 1+c(R-1) .
\end{aligned}
$$

Lemma 20. [1] Let L be a $K$-quasiconformal curve, $z_{1} \in L, z_{2}, z_{3} \in \Omega \cap\left\{z:\left|z-z_{1}\right| \leq\right.$ $\left.d\left(z_{1}, L_{r_{0}}\right)\right\} ; w_{j}=\Phi\left(z_{j}\right), j=1,2,3$. Then

a) The statements $\left|z_{1}-z_{2}\right| \preceq\left|z_{1}-z_{3}\right|$ and $\left|w_{1}-w_{2}\right| \preceq\left|w_{1}-w_{3}\right|$ are equivalent. So are $\left|z_{1}-z_{2}\right| \asymp\left|z_{1}-z_{3}\right|$ and $\left|w_{1}-w_{2}\right| \asymp\left|w_{1}-w_{3}\right|$.

b) If $\left|z_{1}-z_{2}\right| \preceq\left|z_{1}-z_{3}\right|$, then

$$
\left|\frac{w_{1}-w_{3}}{w_{1}-w_{2}}\right|^{\varepsilon} \preceq\left|\frac{z_{1}-z_{3}}{z_{1}-z_{2}}\right| \preceq\left|\frac{w_{1}-w_{3}}{w_{1}-w_{2}}\right|^{c},
$$

where $\varepsilon<1, c>1 ; L_{r_{0}}:=\left\{\zeta:|\varphi(\zeta)|=r_{0}, 0<r_{0}<1\right\}$ and $r_{0}:=r_{0}(G)$ is a constant, depending on $G$.

Lemma 21. Let $G$ be a $k$-quasidisk for some $0 \leq k<1$. Then

$$
\left|\Psi\left(w_{1}\right)-\Psi\left(w_{2}\right)\right| \succeq\left|w_{1}-w_{2}\right|^{1+k},
$$

for all $w_{1}, w_{2} \in \bar{\Omega}^{\prime}$.

This fact is derived to appropriate the results for the estimation $\left|\Psi^{\prime}(\tau)\right|$ (see, [22, p. 287, Lemma 9.9] and [10, Th.2.8]).

The following two Lemmas 22 and 24 we use to prove Theorems 13-16.

Lemma 22. Let $L$ be a K-quasiconformal curve. For arbitrary $R>1$, there exist a numbers $\rho_{1}, \rho_{2}: \rho_{1}<\rho_{2}$, and $\rho_{3}, \rho_{4}: \rho_{3}<\rho_{4}$ such that the following conditions are satisfied:
1) $\bar{G}_{\rho_{1}}^{*} \subseteq \bar{G}, \bar{G} \subseteq \bar{G}_{\rho_{2}}^{*}$ and $\bar{G}_{\rho_{3}}^{*} \subseteq \bar{G}_{R}, \bar{G}_{R} \subseteq \bar{G}_{\rho_{3}}^{*}$

2) $\rho_{1}-1 \asymp \rho_{2}-1 \asymp \rho_{3}-1 \asymp \rho_{4}-1 \asymp R-1$. 
Proof. We consider the four numbers $\rho_{1}, \rho_{2}, \rho_{3}, \rho_{4}$ where $\rho_{1}<\rho_{2}, \rho_{3}<\rho_{4}$ such that

$$
\begin{aligned}
& \bar{G}_{\rho_{1}}^{*} \subseteq \bar{G}, \bar{G}_{R} \subseteq \bar{G}_{\rho_{4}}^{*} \\
& \bar{G} \subseteq \bar{G}_{\rho_{2}}^{*}, \bar{G}_{\rho_{3}}^{*} \subseteq \bar{G}_{R},
\end{aligned}
$$

and we show that the numbers $\rho_{1}, \rho_{2}, \rho_{3}, \rho_{4}$ can be chosen to satisfy the following conditions:

$$
\begin{aligned}
& \rho_{1}-1 \asymp \rho_{4}-1 \asymp R-1 ; \\
& \rho_{2}-1 \asymp \rho_{3}-1 \asymp R-1 .
\end{aligned}
$$

The validity of the relation (3.3) with (3.5) was proved in [3]. We verify the validity of the relation (3.4) with (3.6). Let $\rho_{2}$ be an arbitrary number satisfying (3.4) and let $z \in L^{*}, \widetilde{z}=y(z)$. For $\rho_{2}>1, \rho_{3}>1$ and $R>1$, define points $z_{1} \in L$, $z_{2} \in L_{\rho_{2}}^{*}, z_{3} \in L_{\rho_{3}}^{*}$ and $z_{R} \in L_{R}$ as follows: $d(z, L)=\left|z-z_{1}\right|, d\left(z, L_{\rho_{2}}^{*}\right)=\left|z-z_{2}\right|$, $d\left(z, L_{\rho_{3}}^{*}\right)=\left|z-z_{3}\right|$ and $d\left(z, L_{R}\right)=\left|z-z_{R}\right|$, respectively. According to (3.2), there exists $c_{1}, c_{2}$, independent from $z$ and $R$, such that

$$
\begin{aligned}
c_{1} d\left(z_{1}, L_{R}\right) & \leq d(z, L) \leq c_{2} d\left(z_{1}, L_{R}\right) \\
c_{1} d\left(z, L_{\rho_{2}}^{*}\right) & \leq d(z, L) \leq c_{2} d\left(z, L_{\rho_{2}}^{*}\right)
\end{aligned}
$$

Since $L^{*}$ is a quasiconformal, applying Lemma 20 to the function $\Phi_{R}$ we obtain:

$$
\left|\frac{z-z_{2}}{z-z_{1}}\right| \geq c_{3}\left|\frac{\Phi_{R}(z)-\Phi_{R}\left(z_{2}\right)}{\Phi_{R}(z)-\Phi_{R}\left(z_{1}\right)}\right|^{\varepsilon_{1}} \geq c_{4}\left(\frac{\rho_{2}-1}{\left|\Phi_{R}(z)-\Phi_{R}\left(z_{2}\right)\right|}\right)^{\varepsilon_{1}}
$$

and we get:

$$
\left|z-z_{1}\right| \leq c_{4}^{-1}\left(\frac{\left|\Phi_{R}(z)-\Phi_{R}\left(z_{2}\right)\right|}{\rho_{2}-1}\right)^{\varepsilon_{1}}\left|z-z_{2}\right| .
$$

On the other hand, we can extend the function $\Phi_{R}$ to a quasiconformal mapping $\widetilde{\Phi}_{R}$ of the whole complex plane onto itself. Then, using the $D$-property of the $\widetilde{\Phi}_{R}[12$, p.18] and (3.7) we have:

$$
\left|z-z_{2}\right| \leq c_{5}\left|z-z_{1}\right| \leq c_{5} \cdot c_{2}\left|z-z_{R}\right|, z_{R} \in L_{R}
$$

According to Lemma 20 (written for $\Phi_{R}$ ), we get:

$$
\left|\Phi_{R}(z)-\Phi_{R}\left(z_{2}\right)\right| \leq c_{6}\left|\Phi_{R}(z)-\Phi_{R}\left(z_{R}\right)\right| \leq c_{7}(R-1) .
$$

Then, from (3.8), we obtain:

$$
\left|z-z_{1}\right| \leq c_{4}^{-1}\left(\frac{c_{7}(R-1)}{\rho_{2}-1}\right)^{\varepsilon_{1}}\left|z-z_{2}\right|
$$

By taking:

$$
\rho_{2}=1+c_{8}(R-1)
$$

with $c_{8}=2 c_{7} \cdot c_{4}^{-\varepsilon_{1}^{-1}}$, which leads to (3.4) and (3.6). 
Now, we will define $\rho_{3}$. Applying Lemma 20 to $\Phi_{R}$ we get:

$$
\left|\frac{z-z_{R}}{z-z_{3}}\right| \geq c_{9}\left|\frac{\Phi_{R}(z)-\Phi_{R}\left(z_{R}\right)}{\Phi_{R}(z)-\Phi_{R}\left(z_{3}\right)}\right|^{\varepsilon_{1}} \geq c_{10}\left|\frac{\Phi_{R}(z)-\Phi_{R}\left(z_{R}\right)}{\rho_{3}-1}\right|^{\varepsilon_{1}},
$$

and we obtain:

$$
\left|z-z_{3}\right| \leq c_{10}^{-1}\left(\frac{\rho_{3}-1}{\left|\Phi_{R}(z)-\Phi_{R}\left(z_{R}\right)\right|}\right)^{\varepsilon_{1}}\left|z-z_{R}\right|
$$

Since $\left|z-z_{R}\right| \geq d\left(L, L_{R}\right)=: d\left(\widehat{z}, L_{R}\right)$, where $\widehat{z} \in L$, then $\left|\Phi_{R}(z)-\Phi_{R}\left(z_{R}\right)\right| \geq$ $c_{11}\left|\Phi_{R}(\widehat{z})-\Phi_{R}\left(z_{R}\right)\right| \geq c_{12}(R-1)$, and from (3.10) we have:

$$
\left|z-z_{3}\right| \leq c_{10}^{-1}\left(\frac{\rho_{3}-1}{c_{12}(R-1)}\right)^{\varepsilon_{1}}\left|z-z_{R}\right|
$$

By choosing

$$
\rho_{3}=1+c_{13}(R-1)
$$

with $c_{13}=\frac{1}{2} c_{12} \cdot c_{10}^{\varepsilon_{1}^{-1}}$, we see the second part of (3.4) and (3.6) are also satisfied.

Let $\left\{z_{j}\right\}_{j=1}^{m}$ be a fixed system of the points on $L$ and the weight function $h(z)$ is defined as the following:

$$
h(z)=\prod_{j=1}^{m}\left|z-z_{j}\right|^{\gamma_{j}}, \gamma_{j}>-2, j=\overline{1, m}, z \in G_{R}
$$

Lemma 23. [3] Let L be a $K$-quasiconformal curve; $h(z)$ is defined as in (3.12). Then, for arbitrary $P_{n}(z) \in \wp_{n}$, any $R>1$ and $n \in \mathbb{N}$, we have

$$
\left\|P_{n}\right\|_{A_{p}\left(h, G_{R}\right)} \preceq[1+c(R-1)]^{n+\frac{1}{p}}\left\|P_{n}\right\|_{A_{p}(h, G)}, p>0 .
$$

Lemma 24. Let $L$ be a $K$-quasiconformal curve; $L^{*}:=y\left(L_{R}\right), R=1+\frac{1}{n}$; $\rho:=1+c_{1}(R-1)$. Then, for any fixed $\varepsilon \in(0,1)$ there exists level curve $L_{1+\varepsilon(\rho-1)}^{*}$ such that for $P_{n}(z) \in \wp_{n}, n \in \mathbb{N}$, we have

$$
\left\|P_{n}\right\|_{\mathfrak{L}_{p}\left(\left|\Phi_{R}^{\prime}\right|^{-1}, L_{1+\varepsilon(\rho-1)}^{*}\right)} \preceq n^{\frac{1}{p}}\left\|P_{n}\right\|_{A_{p}(G)}, p>0 .
$$

Proof. Case 1.

For arbitrary fixed $R>1$, let $\rho_{1}:=\sup \left\{\rho=1+c_{1}(R-1): \bar{G}_{\rho}^{*} \subseteq G\right\}$, and, without loss of generality, we may take $\varepsilon=\frac{1}{2}$ ant $R_{1}:=1+\frac{\rho_{1}-1}{2}$. Let us set:

$$
f_{n}(w):=P_{n}\left(\Psi_{R}(w)\right) \cdot\left(\Psi_{R}^{\prime}(w)\right)^{\frac{2}{p}} .
$$


Then,

$$
\begin{aligned}
J_{R_{1}}^{p} & :=\int_{L_{R_{1}}^{*}}\left|P_{n}(z)\right|^{p} \frac{|d z|}{\left|\Phi_{R}^{\prime}(z)\right|} \\
& =\int_{|w|=R_{1}}\left|P_{n}\left(\Psi_{R}(w)\right)\left(\Psi_{R}^{\prime}(w)\right)^{\frac{2}{p}}\right|^{p}|d w| \\
& =\int_{|w|=R_{1}}\left|f_{n, p}(w)\right|^{p}|d w|
\end{aligned}
$$

where $f_{n, p}(w):=P_{n}\left(\Psi_{R}(w)\right)\left(\Psi_{R}^{\prime}(w)\right)^{\frac{2}{p}},|w|=R_{1}$. Now, we separate the circle $|t|=R_{1}$ to $n$ equal parts $\delta_{n}$ with mes $\delta_{n}=\frac{2 \pi R_{1}}{n}$ and by applying the mean value theorem to the integral $A_{n}^{1}$, we get:

$$
\begin{aligned}
& J_{R_{1}}^{p}=\int_{|t|=R_{1}}\left|f_{n}(w)\right|^{p}|d w| \\
= & \sum_{k=1}^{n} \int_{\delta_{k}}\left|f_{n}(w)\right|^{p}|d w|=\sum_{k=1}^{n}\left|f_{n}\left(t_{k}^{\prime}\right)\right|^{p} \operatorname{mes} \delta_{k}, \quad t_{k}^{\prime} \in \delta_{k} .
\end{aligned}
$$

On the other hand, by applying mean value estimation

$$
\left|f_{n}\left(t_{k}^{\prime}\right)\right|^{p} \leq \frac{1}{\pi\left(\left|t_{k}^{\prime}\right|-1\right)^{2}} \iint_{\left|\xi-t_{k}^{\prime}\right|<\left|t_{k}^{\prime}\right|-1}\left|f_{n}(\xi)\right|^{p} d \sigma_{\xi}
$$

we obtain:

$$
J_{R_{1}}^{p} \preceq \sum_{k=1}^{n} \frac{m e s \delta_{k}}{\pi\left(\left|t_{k}^{\prime}\right|-1\right)^{2}} \iint_{\left|\xi-t_{k}^{\prime}\right|<\left|t_{k}^{\prime}\right|-1}\left|f_{n}(\xi)\right|^{p} d \sigma_{\xi}, t_{k}^{\prime} \in \delta_{k} .
$$

By taking into account, at most two of the discs with origin at the points $t_{k}^{\prime}$ are intersecting, we have:

$$
J_{R_{1}}^{p} \preceq \frac{\operatorname{mes} \delta_{1}}{\left(\left|t_{1}^{\prime}\right|-1\right)^{2}} \iint_{1<|\xi|<\rho_{1}}\left|f_{n}(\xi)\right|^{p} d \sigma_{\xi} \preceq n \cdot \iint_{1<|\xi|<\rho_{1}}\left|f_{n}(\xi)\right|^{2} d \sigma_{\xi} .
$$

According to (23), for $J_{R_{1}}^{p}$, we get:

$$
J_{R_{1}}^{p} \preceq n \iint_{G_{\rho_{1}}^{*} \backslash G^{*}}\left|P_{n}(z)\right|^{p} d \sigma_{z} \preceq n \cdot\left\|P_{n}\right\|_{A_{p}(G)}^{p} .
$$


Case 2. Let us now take $R_{2}:=1+\frac{\rho_{2}-1}{2}$, where $\rho_{2}:=\sup \left\{\rho=1+c_{2}(R-1)\right)$ : $\left.G_{\rho}^{*} \subseteq \bar{G}_{R}\right\}$, similarly to the previous case, we obtain:

$$
\begin{aligned}
J_{R_{2}}^{p} & :=\int_{L_{R_{2}}^{*}}\left|P_{n}(z)\right|^{p} \frac{|d z|}{\left|\Phi_{R}^{\prime}(z)\right|} \\
& \preceq n \iint_{G_{\rho_{2}}^{*} \backslash G^{*}}\left|P_{n}(z)\right|^{p} d \sigma_{z} \preceq n\left\|P_{n}\right\|_{A_{p}\left(G_{R}\right)}^{p} .
\end{aligned}
$$

Setting $R=1+\frac{1}{n}$ and applying Lemma 23 for $h(z) \equiv 1$, we have:

$$
J_{R_{2}}^{p} \preceq n\left\|P_{n}\right\|_{A_{p}(G)}^{p} .
$$

Combining (3.15)-(3.17), we have proved the estimation (3.14):

$$
\left\|\frac{P_{n}}{\Phi_{R}^{\prime}}\right\|_{\mathfrak{L}_{p}\left(L_{1+\varepsilon\left(\rho_{1}-1\right)}^{*}\right)} \preceq n^{\frac{1}{p}}\left\|P_{n}\right\|_{A_{p}(G)} .
$$

\section{Proof of Theorems}

\subsection{Proof of Theorem A}

Proof. For the arbitrary polynomials $P_{n} \in \wp_{n}$ and in particular, for Bergman polynomials (i.e. orthonormal polynomials over the region $K_{n}(z):\left\|K_{n}\right\|_{A_{2}(h, G)}=1$ ) in [5, Th.2.1 and Th.5.1] the following is proved:

Theorem. Let $G$ be bounded by $k$-quasidisk for some $0 \leq k<1$ and let the weight function $h(z)$ be defined by (3.12). Then, for any $P_{n} \in \wp_{n}, n \in \mathbb{N}$, and every point $z_{j} \in L, j=1,2, \ldots, m$,

$$
\left|P_{n}\left(z_{j}\right)\right| \preceq n^{\frac{\left(2+\gamma_{j}\right)(1+k)}{p}}\left\|P_{n}\right\|_{A_{p}(h, G)} .
$$

Putting $h(z) \equiv 1$, i.e. $\gamma_{j}=0, j=1,2, \ldots, m$, we obtain the proof.

\subsection{Proof of Theorem 2}

Proof. Let $R=1+\frac{1}{n}$. For the sufficiently small $\varepsilon_{1}>0$, let us set: $R_{1}:=1+\varepsilon_{1}$ $(R-1)$. Let $\left\{\zeta_{j}\right\}, 1 \leq j \leq m \leq n$, zeros of $P_{n}(z)$ lying on $\Omega$ and let

$$
B(z):=\prod_{j=1}^{m} B_{j}(z)=\prod_{j=1}^{m} \frac{\Phi(z)-\Phi\left(\zeta_{j}\right)}{1-\overline{\Phi\left(\zeta_{j}\right)} \Phi(z)}
$$

denote a Blashke function with respect of zeros of $P_{n}(z)$. For any $p>0$ and $z \in \Omega$ let us set:

$$
\widetilde{F}_{n, p}(z):=\left(\frac{P_{n}(z)}{B(z) \Phi^{n+1}(z)}\right)^{\frac{p}{2}} .
$$


Cauchy integral representation for a region $\Omega$ is given as:

$$
\widetilde{F}_{n, p}(z)=-\frac{1}{2 \pi i} \int_{L_{R_{1}}} \widetilde{F}_{n, p}(\zeta) \frac{d \zeta}{\zeta-z}, z \in \Omega_{R_{1}} .
$$

Since $|B(\zeta)|=1$, for $\zeta \in L$, then, for arbitrary $\varepsilon, 0<\varepsilon<\varepsilon_{1}$, there exists a circle $|w|=1+\frac{\varepsilon}{n}$, such that for any $j=1,2, \ldots, m$ the following is satisfied:

$$
\mid B_{j}\left(\Psi_{R}(w) \mid>1-\varepsilon .\right.
$$

Then, $|B(\zeta)|>(1-\varepsilon)^{m} \succeq 1$. On the other hand, $|\Phi(\zeta)|=R_{1}>1$, for $\zeta \in L_{R_{1}}$. Therefore, for any $z \in \Omega_{R_{1}}$ we have:

$$
\left|\widetilde{F}_{n, p}(z)\right|=\frac{1}{2 \pi} \int_{L_{R_{1}}}\left|\widetilde{F}_{n, p}(\zeta)\right| \frac{|d \zeta|}{|\zeta-z|} \preceq \frac{1}{d(z, L)} \int_{L_{R_{1}}}\left|P_{n}(\zeta)\right|^{\frac{p}{2}}|d \zeta| .
$$

By applying the Hölder inequality from Lemma 24, we get:

$$
\begin{aligned}
\int_{L_{R_{1}}}\left|P_{n}(\zeta)\right|^{\frac{p}{2}}|d \zeta| & =\int_{|w|=R_{1}}\left|P_{n}(\Psi(w))\right|^{\frac{p}{2}}\left|\Psi^{\prime}(w)\right||d w| \\
& \leq \sqrt{2 \pi R_{1}}\left(\int_{|w|=R_{1}}\left|P_{n}(\Psi(w))\right|^{p}\left|\Psi^{\prime}(w)\right|^{2}|d w|\right)^{\frac{1}{2}} \\
& \preceq\left(n\left\|P_{n}\right\|_{A_{p}(G)}^{p}\right)^{\frac{1}{2}} .
\end{aligned}
$$

Then,

$$
\left(\int_{L_{R_{1}}}\left|P_{n}(\zeta)\right|^{\frac{p}{2}}|d \zeta|\right)^{\frac{2}{p}} \preceq n^{\frac{1}{p}}\left\|P_{n}\right\|_{A_{p}(G)} .
$$

From (4.2) and (4.3), we obtain:

$$
\begin{aligned}
\left|P_{n}(z)\right| & \preceq \frac{n^{\frac{1}{p}}}{d^{\frac{2}{p}}\left(z, L_{R_{1}}\right)}|B(z)||\Phi(z)|^{n+1}\left\|P_{n}\right\|_{A_{p}(G)} \\
& =\left(\frac{\sqrt{n}}{d\left(z, L_{R_{1}}\right)}\right)^{\frac{2}{p}}|B(z)|\left\|P_{n}\right\|_{A_{p}(G)}|\Phi(z)|^{n+1}, z \in \Omega_{R_{1}} .
\end{aligned}
$$

The function $B(z)$ is analytic in $\Omega$, continuous on $\bar{\Omega}$ and $|B(z)|=1$ on $L$. Then, according to the maximum modulus principle, we get

$$
|B(z)|<1, z \in \Omega_{R_{1}},
$$

and, then the proof is complete. 


\subsection{Proof of Theorem 6}

Proof. Let $R_{1}=1+\frac{1}{2 n}$. For any $p \geq 1$ and $z \in \Omega_{R_{1}}$ let us set:

$$
G_{n, p}(z):=\left(\frac{P_{n}(z)}{\Phi^{n+1}(z)}\right)^{p} .
$$

The Cauchy integral representation for unbounded region $\Omega_{R_{1}}$ gives

$$
G_{n, p}(z)=-\frac{1}{2 \pi i} \int_{L_{R_{1}}} G_{n, p}(\zeta) \frac{d \zeta}{\zeta-z}, z \in \Omega_{R_{1}}
$$

Then

$$
\left|G_{n, p}(z)\right|=\frac{1}{2 \pi} \int_{L_{R_{1}}}\left|G_{n, p}(\zeta)\right| \frac{|d \zeta|}{|\zeta-z|} \leq \frac{1}{2 \pi d\left(z, L_{R_{1}}\right)} \int_{L_{R_{1}}}\left|P_{n}(\zeta)\right|^{p}|d \zeta| .
$$

By applying the Hölder inequality, we get:

$$
\begin{aligned}
\left|G_{n, p}(z)\right| & \preceq \frac{1}{d\left(z, L_{R_{1}}\right)} \int_{L_{R_{1}}}\left|P_{n}(\zeta)\right|^{p}|d \zeta| \\
& =\frac{1}{d\left(z, L_{R_{1}}\right)} \int_{|w|=R_{1}}\left|P_{n}(\Psi(w)) \cdot\left(\Psi^{\prime}(w)\right)^{\frac{2}{p}}\right|^{p} \frac{|d w|}{\left|\Psi^{\prime}(w)\right|} \\
& \leq \frac{1}{d\left(z, L_{R_{1}}\right)} \cdot \sup _{|w|=R_{1}} \frac{1}{\left|\Psi^{\prime}(w)\right|} \int_{|w|=R_{1}}\left|f_{n, p}(w)\right|^{p}|d w|
\end{aligned}
$$

where $f_{n, p}(w):=P_{n}(\Psi(w))(\Psi(w))^{\frac{2}{p}}$. Thus, taking into account that the estimation for the $\left|\Psi^{\prime}\right|$ (see, for instance, [10, Th.2.8]) and by applying Lemma 24, we get

$$
\left|G_{n, p}(z)\right| \preceq \frac{1}{d\left(z, L_{R_{1}}\right)} \cdot \sup _{|w|=R_{1}} \frac{|w|-1}{d(\Psi(w), L)} \cdot n\left\|P_{n}\right\|_{A_{p}(G)}^{p} .
$$

Now, since $G \in \widetilde{Q}_{\alpha}$, we get

$$
d(\Psi(w), L) \succeq(|w|-1)^{\frac{1}{\alpha}}
$$

Hence, we have:

$$
\begin{aligned}
\left|G_{n, p}(z)\right| & \preceq \frac{1}{d\left(z, L_{R_{1}}\right)} \cdot \sup _{|w|=R_{1}} \frac{1}{(|w|-1)^{\frac{1}{\alpha}-1}} \cdot n\left\|P_{n}\right\|_{A_{p}(G)}^{p} \\
& \preceq \frac{n^{\frac{1}{\alpha}}}{d\left(z, L_{R_{1}}\right)}\left\|P_{n}\right\|_{A_{p}(G)}^{p} .
\end{aligned}
$$

By (4.4), we obtain:

$$
\left|P_{n}(z)\right| \preceq\left(\frac{n^{\frac{1}{\alpha}}}{d\left(z, L_{R_{1}}\right)}\right)^{\frac{1}{p}}\left\|P_{n}\right\|_{A_{p}(G)}\left|\Phi^{n+1}(z)\right|, z \in \Omega_{R_{1}}, p \geq 1 .
$$




\subsection{Proof of Theorem 9}

Proof. The proof of the Theorem 9 is identical to the proof of the Theorem 6 . In this case, we consider the following:

$$
G_{n, 1}(z):=\frac{P_{n}(z)}{\Phi^{n+1}(z)}
$$

The Cauchy integral representation is given as

$$
G_{n, 1}(z)=-\frac{1}{2 \pi i} \int_{L_{R_{1}}} G_{n, 1}(\zeta) \frac{d \zeta}{\zeta-z}, z \in \Omega_{R_{1}} .
$$

Then, analogously to (4.5) and (4.7), we have:

$$
\begin{gathered}
\left|G_{n, 1}(z)\right| \preceq \frac{1}{d\left(z, L_{R_{1}}\right)} \int_{L_{R_{1}}}\left|P_{n}(\zeta)\right||d \zeta| \\
=\frac{1}{d\left(z, L_{R_{1}}\right)} \int_{|w|=R_{1}}\left|P_{n}(\Psi(w))\left(\Psi^{\prime}(w)\right)^{\frac{2}{p}}\right| \frac{|d w|}{\left|\Psi^{\prime}(w)\right|^{\frac{2}{p}-1}} \\
\leq \frac{1}{d\left(z, L_{R_{1}}\right)}\left(\int_{|w|=R_{1}}\left|f_{n, p}(w)\right|^{p}|d w|\right)^{\frac{1}{p}} \cdot\left(\int_{|w|=R_{1}}\left|\Psi^{\prime}(w)\right|^{\left(1-\frac{2}{p}\right) q}|d w|\right)^{\prime}
\end{gathered}
$$

where $f_{n, p}(w):=P_{n}(\Psi(w))(\Psi(w))^{\frac{2}{p}}$ and $\frac{1}{p}+\frac{1}{q}=1$. Since $\left(1-\frac{2}{p}\right) q=2-q$, then, for any $p>1$, we get:

$$
\begin{aligned}
\left|G_{n, 1}(z)\right| & \preceq \frac{1}{d\left(z, L_{R_{1}}\right)}\left(\int_{|w|=R_{1}}\left|f_{n, p}(w)\right|^{p}|d w|\right)^{\frac{1}{p}}\left(\int_{|w|=R_{1}}\left|\Psi^{\prime}(w)\right|^{2-q}|d w|\right)^{\frac{1}{q}} \\
& =: A_{n, p} \cdot B_{n, q} .
\end{aligned}
$$

For the integral $A_{n, p}$, from the Lemma 24, we obtain:

$$
A_{n, p} \preceq n^{\frac{1}{p}}\left\|P_{n}\right\|_{A_{p}(G)} .
$$

For the integral $B_{n, q}$, by taking into account of the estimation for the $\left|\Psi^{\prime}\right|$ ( [10, Th.2.8]), we get:

$$
B_{n, q}^{q}:=\int_{|w|=R_{1}}\left|\Psi^{\prime}(w)\right|^{2-q}|d w| \asymp \int_{|w|=R_{1}}\left(\frac{d(\Psi(w), L)}{|w|-1}\right)^{2-q}|d w| .
$$

Let $1<q<2$. Since $G \in Q_{\alpha}^{\beta}$, for $z^{*} \in L$ such that $d(\Psi(w), L)=\left|\Psi(w)-z^{*}\right|$ and $w^{*}=\Phi\left(z^{*}\right)$, we have:

$$
d(\Psi(w), L) \preceq\left|w-w^{*}\right|^{\beta} \preceq(|w|-1)^{\beta} .
$$


Then, according to Lemma 20, from (4.12), we obtain:

$$
\begin{aligned}
B_{n, q}^{q} & \preceq \int_{|w|=R_{1}}\left(\frac{(|w|-1)^{\beta}}{|w|-1}\right)^{2-q}|d w| \preceq \int_{|w|=R_{1}} \frac{|d w|}{(|w|-1)^{(1-\beta)(2-q)}} \\
& \preceq \frac{1}{\left(R_{1}-1\right)^{(1-\beta)(2-q)}} .
\end{aligned}
$$

Since $R_{1}:=1+\frac{1}{2 n}$, in this case, from Lemma 22 we have:

$$
B_{n, q} \preceq n^{\frac{(1-\beta)(2-q)}{q}} .
$$

Let $q>2$. Let us put $d\left(\Psi_{R}(w), L\right):=\left|\widetilde{z}-z^{*}\right|, \widetilde{z} \in L_{R_{1}}$ and $\widetilde{w}:=\Phi(\widetilde{z})$. Since $G \in Q_{\alpha}^{\beta}$, according to (4.7), we get $\left|\widetilde{z}-z^{*}\right| \succeq\left|\widetilde{w}-w^{*}\right|^{\frac{1}{\alpha}}$. In this case, from (4.12) we obtain:

$$
\begin{aligned}
B_{n, q}^{q} & \asymp \int_{|w|=R_{1}}\left(\frac{|w|-1}{d(\Psi(w), L)}\right)^{q-2}|d w| \preceq \int_{|w|=R_{1}}\left(\frac{|w|-1}{\left|\widetilde{w}-w^{*}\right|^{\frac{1}{\alpha}}}\right)^{q-2}|d w| \\
& \preceq \int_{|w|=R_{1}} \frac{|d w|}{\left|\widetilde{w}-w^{*}\right|^{\left(\frac{1}{\alpha}-1\right)(q-2)} \preceq n^{\left(\frac{1}{\alpha}-1\right)(q-2)-1},\left(\frac{1}{\alpha}-1\right)(q-2)<1 .}
\end{aligned}
$$

Then,

$$
B_{n, q} \preceq n^{\frac{\left(\frac{1}{\alpha}-1\right)(q-2)-1}{q}}, q<1+\frac{1}{1-\alpha} .
$$

By combining (4.9)-(4.14), we obtain:

$$
\left|P_{n}(z)\right| \preceq \frac{|\Phi(z)|^{n+1}}{d\left(z, L_{R_{1}}\right)}\left\|P_{n}\right\|_{A_{p}(G)}\left\{\begin{array}{cc}
n^{\frac{1}{\alpha}\left(\frac{2}{p}-1\right)}, & 2-\alpha<p<2 \\
\sqrt{n}, & p=2, \\
n^{(1-\beta)\left(1-\frac{2}{p}\right)} & \beta>0, p>2,
\end{array}, z \in \Omega_{R_{1}}\right.
$$

\subsection{Proof of Theorem 11}

Proof. Since $L$ is a quasiconformal, we conclude that any $L_{R}, R>1$, is also quasiconformal. Therefore, we construct the $c_{1}(K)$-quasiconformal reflection $y_{R}(z)$, $y_{R}(0)=\infty$, across $L_{R}$ such that $y_{R}\left(G_{R}\right)=\Omega_{R}, y_{R}\left(\Omega_{R}\right)=G_{R}$ and $y_{R}($.$) fixes$ the points of $L_{R}$ that satisfies conditions (3.1) described for $y_{R}(z)$. By using this constructed $y_{R}(z)$, we write the following integral representations for $G_{n, 1}(z)$ :

$$
\begin{aligned}
G_{n, 1}(z) & =-\frac{1}{2 \pi i} \int_{L_{R}} G_{n, 1}(\zeta) \frac{d \zeta}{\zeta-z} \\
& =-\frac{1}{2 \pi i} \int_{L_{R}} \frac{g(\zeta) d \zeta}{\Phi^{n+1}\left(y_{R}(\zeta)\right)}, z \in \Omega_{R},
\end{aligned}
$$


where $g(\zeta):=\frac{P_{n}(\zeta)}{\zeta-z}$. The Green formula is given as:

$$
\begin{aligned}
G_{n, 1}(z) & =-\frac{1}{\pi} \iint_{G_{R}}\left[\frac{g(\zeta)}{\Phi^{n+1}\left(y_{R}(\zeta)\right)}\right]_{\bar{\zeta}} d \sigma_{\zeta} \\
& =\frac{n+1}{\pi} \iint_{G_{R}} g(\zeta) \frac{\Phi^{\prime}\left(y_{R}(\zeta)\right)}{\Phi^{n+2}\left(y_{R}(\zeta)\right)} y_{R}, \bar{\zeta} d \sigma_{\zeta}
\end{aligned}
$$

Hence, by applying the Hölder inequality, we get:

$$
\begin{gathered}
\left|G_{n, 1}(z)\right|^{2} \leq\left(\frac{n+1}{\pi}\right)^{2} \iint_{G_{R}}|g(\zeta)|^{2} d \sigma_{\zeta} \cdot \iint_{G_{R}}\left|\frac{\Phi^{\prime}\left(y_{R}(\zeta)\right)}{\Phi^{n+2}\left(y_{R}(\zeta)\right)} y_{R}, \bar{\zeta}\right|^{2} d \sigma_{\zeta} \\
=: J_{1} \cdot J_{2}, z \in \Omega_{R} .
\end{gathered}
$$

Since $z \in \Omega_{R}$, then for the integral $J_{1}$ we have:

$$
\begin{aligned}
J_{1} & :=\iint_{G_{R}}|g(\zeta)|^{2} d \sigma_{\zeta} \\
= & \iint_{G_{R}}\left|\frac{P_{n}(\zeta)}{\zeta-z}\right|^{2} d \sigma_{\zeta} \leq \frac{1}{d^{2}\left(z, L_{R}\right)} \iint_{G_{R}}\left|P_{n}(\zeta)\right|^{2} d \sigma_{\zeta} .
\end{aligned}
$$

For the estimation of the integral $J_{2}$, we note that if $J_{y_{R}}:=\left|y_{R, \zeta}\right|^{2}-\left|y_{R, \bar{\zeta}}\right|^{2}$ is Jacobian of the reflection $y_{R}(\zeta)$, which satisfies the conditions (3.1) and is described for $y_{R}(z)$.

$$
\left|y_{R, \bar{\zeta}}\right|^{2} \leq \frac{J_{y_{R}}}{1-k_{1}^{2}}
$$

where $k_{1}:=\frac{c_{1}(K)-1}{c_{1}(K)+1}<1$ and $c_{1}(K)$ is a coefficient of quasiconformality of the reflection $y_{R}(\zeta)$. Then, after changing of the variable $t=y_{s}^{*}(\zeta)$ and $w=\Phi(z)$, we find

$$
\begin{gathered}
J_{2}:=\iint_{G_{R}}\left|\frac{\Phi^{\prime}\left(y_{R}(\zeta)\right)}{\Phi^{n+2}\left(y_{R}(\zeta)\right)} y_{R}, \bar{\zeta}\right|^{2} d \sigma_{\zeta} \\
\leq \frac{1}{1-k_{1}^{2}} \iint_{G_{R}}\left|\frac{\Phi^{\prime}\left(y_{R}(\zeta)\right)}{\Phi^{n+2}\left(y_{R}(\zeta)\right)}\right|^{2} J_{y_{R}} d \sigma_{\zeta}=\frac{1}{1-k_{1}^{2}} \iint_{\Omega_{R}}\left|\frac{\Phi^{\prime}(t)}{\Phi^{n+2}(t)}\right|^{2} d \sigma_{t} \\
=\frac{1}{1-k_{1}^{2}} \iint_{|w|>R} \frac{d \sigma_{w}}{|w|^{2 n+4}}=\frac{\pi}{n+1} \cdot \frac{1}{1-k_{1}^{2}} \cdot \frac{1}{R^{2 n+2}} \leq \frac{\pi}{n+1} \cdot \frac{1}{1-k_{1}^{2}} .
\end{gathered}
$$

By combining (4.17)-(4.19), we get:

$$
\left|P_{n}(z)\right| \leq \sqrt{\frac{n+1}{\pi\left(1-k_{1}^{2}\right)}} \frac{1}{d(z, L)}\left\|P_{n}\right\|_{A_{2}\left(G_{R}\right)}\left|\Phi^{n+1}(z)\right|, R>1, z \in \Omega_{R} .
$$




\subsection{Proof of Corollary 12.}

Proof. Let $z \in F \Subset \Omega$ be a arbitrary fixed point. Let us set: $d\left(z, L_{1+\frac{1}{n}}\right):=\left|z-\zeta_{n}\right|$, $\zeta_{n} \in L_{1+\frac{1}{n}}, d(z, L):=|z-\zeta|, \zeta \in L$ and $w:=\Phi(z), t_{n}:=\Phi\left(\zeta_{n}^{n}\right), t:=\Phi(\zeta)$. Obviously, $\zeta_{n} \rightarrow \zeta$, at $n \rightarrow \infty$. Hence, $\left|t-t_{n}\right| \leq \varepsilon_{n}|w-t|$, where $\varepsilon_{n} \rightarrow 0$ at $n \rightarrow \infty$. Then, we find

$$
\begin{aligned}
\left|w-t_{n}\right| & \geq|w-t|-\left|t-t_{n}\right| \geq|w-t|-\varepsilon_{n}|w-t| \\
& =\left(1-\varepsilon_{n}\right)|w-t| \geq \frac{1}{2}|w-t| .
\end{aligned}
$$

According to Lemma 20, we obtain $d\left(z, L_{1+\frac{1}{n}}\right) \succeq d(z, L)$. Consequently, from (4.20), we get:

$$
\left|P_{n}(z)\right| \preceq \frac{\sqrt{n}}{d(z, L)}\left\|P_{n}\right\|_{A_{2}(G)}\left|\Phi^{n+1}(z)\right|, z \in F \Subset \Omega .
$$

\subsection{Proof of Theorem 13}

Proof. For arbitrary fixed $s>1$, let $L_{s}^{*}=\left\{z:\left|\Phi_{R}(z)\right|=s\right\}$. According to Lemma 22, we choose $s>1$ such that $L_{s}^{*} \subset \bar{G}$. Then, $\overline{G_{s}^{*}} \subset \bar{G}$ and $\Omega \subset \Omega_{s}^{*}$.

The function

$$
T_{R, n}(z):=\frac{P_{n}(z)}{\Phi_{R}^{n+1}(z)}
$$

is analytic in $\bar{\Omega}^{*}$ and $T_{R, n}(\infty)=0$.

Let $y_{s}^{*}(z), y_{R}(0)=\infty$, is a $c_{2}(K)$-quasiconformal reflection $\left(c_{2}(K)>1\right)$ across $L_{s}^{*}$ such that $y_{s}^{*}\left(G_{s}^{*}\right)=\Omega_{s}^{*}, y_{s}^{*}\left(\Omega_{s}^{*}\right)=G_{s}^{*}$ and $y_{s}($.$) fixed the points of L_{s}^{*}$ that satisfies the conditions (3.1) which are rewritten for $y_{s}^{*}(z)$. If $J_{y_{s}}^{*}=\left|y_{s, z}^{*}\right|^{2}-$ $\left|y_{s, \bar{z}}^{*}\right|^{2}$ is the Jacobian of $y_{s}^{*}($.$) , then the relation$

$$
\left|y_{s, \bar{z}}^{*}\right|^{2} \leq \frac{1}{1-k_{2}^{2}} J_{y_{s}}^{*}
$$

is hold, where $k_{2}=\frac{c_{2}(K)^{2}-1}{c_{2}(K)^{2}+1}<1$.

The Cauchy integral representation for $\bar{\Omega}^{*}$ is given as:

$$
\begin{aligned}
T_{R, n}(z) & =-\frac{1}{2 \pi i} \int_{L_{s}^{*}} T_{R, n}(\zeta) \frac{d \zeta}{\zeta-z} \\
& =-\frac{1}{2 \pi i} \int_{L_{s}^{*}} g(\zeta) \frac{d \zeta}{\Phi_{R}^{n+1}\left(y_{s}^{*}(\zeta)\right)}, z \in \Omega,
\end{aligned}
$$


where $g(\zeta):=\frac{P_{n}(\zeta)}{\zeta-z}$. Green formula gives

$$
\begin{aligned}
T_{R, n}(z) & =-\frac{1}{\pi} \iint_{G_{s}^{*}}\left[\frac{g(\zeta)}{\Phi_{R}^{n+1}\left(y_{R}(\zeta)\right)}\right]_{\bar{\zeta}} d \sigma_{\zeta} \\
& =\frac{n+1}{\pi} \iint_{G_{s}^{*}} g(\zeta) \frac{\Phi_{R}^{\prime}\left(y_{s}^{*}(\zeta)\right) \cdot y_{s}^{*}, \bar{\zeta}}{\Phi_{R}^{n+2}\left(y_{s}^{*}(\zeta)\right)} d \sigma_{\zeta} .
\end{aligned}
$$

Hence, by applying the Hölder inequality, we get:

$$
\begin{aligned}
\left|T_{R, n}(z)\right|^{2} & \leq\left(\frac{n+1}{\pi}\right)^{2} \iint_{G_{s}^{*}}|g(\zeta)|^{2} d \sigma_{\zeta} \\
\times \iint_{G_{s}^{*}}\left|\frac{\Phi_{R}^{\prime}\left(y_{s}^{*}(\zeta)\right) \cdot y_{s}^{*}, \bar{\zeta}}{\Phi_{R}^{n+2}\left(y_{s}^{*}(\zeta)\right)}\right|^{2} d \sigma_{\zeta} & =: \widetilde{J_{1}} \cdot \widetilde{J_{2}}, z \in \Omega .
\end{aligned}
$$

For the integral $\widetilde{J_{1}}$, we obtain:

$$
\begin{aligned}
{\widetilde{J_{1}}}^{2} & :=\iint_{G_{s}^{*}}|g(\zeta)|^{2} d \sigma_{\zeta} \\
& \leq \frac{1}{d\left(z, L_{s}^{*}\right)} \iint_{G_{s}^{*}}\left|P_{n}(\zeta)\right|^{2} d \sigma_{\zeta} \leq \frac{1}{d(z, L)} \iint_{G}\left|P_{n}(\zeta)\right|^{2} d \sigma_{\zeta} .
\end{aligned}
$$

According to (4.21), after changing of the variable $t=y_{s}^{*}(\zeta)$ and $w=\Phi_{R}(z)$, for integral $\widetilde{J}_{2}$ we find

$$
\begin{aligned}
{\widetilde{J_{2}}}^{2} & :=\iint_{G_{s}^{*}}\left|\frac{\Phi_{R}^{\prime}\left(y_{s}^{*}(\zeta)\right) \cdot y_{s}^{*}, \bar{\zeta}}{\Phi_{R}^{n+2}\left(y_{s}^{*}(\zeta)\right)}\right|^{2} d \sigma_{\zeta} \leq \frac{1}{1-k_{2}^{2}} \iint_{G_{s}^{*}}\left|\frac{\Phi_{R}^{\prime}\left(y_{s}^{*}(\zeta)\right)}{\Phi_{R}^{n+2}\left(y_{s}^{*}(\zeta)\right)}\right|^{2} J_{y_{s}}^{*} d \sigma_{\zeta} \\
& =\frac{1}{1-k_{2}^{2}} \iint_{\Omega_{s}^{*}}\left|\frac{\Phi_{R}^{\prime}(t)}{\Phi_{R}^{n+2}(t)}\right|^{2} d \sigma_{t}=\frac{1}{1-k_{2}^{2}} \iint_{w \mid>s} \frac{d \sigma_{\tau}}{\tau^{2 n+4}}=\frac{1}{1-k_{2}^{2}} \frac{\pi}{n+1} \frac{1}{s^{2 n+2}}
\end{aligned}
$$

Since $s>1$, then

$$
\widetilde{J}_{2} \leq \sqrt{\frac{1}{1-k_{2}^{2}} \frac{\pi}{n+1}}
$$

By combining (4.23)-(4.25), we get:

$$
\left|P_{n}(z)\right| \leq \sqrt{\frac{n+1}{\pi\left(1-k_{2}^{2}\right)}} \frac{1}{d(z, L)}\left\|P_{n}\right\|_{A_{2}(G)}\left|\Phi_{R}(z)\right|^{n+1}, z \in \Omega .
$$

The proof of Theorem 13 is completed. 


\subsection{Proofs of Theorems 13 (in case of $p \neq 2$ ), 15 and 16}

Proof. The proof of Theorems 13 (in case of $p \neq 2$ ), 15 and 16 are similar to the proof of Theorems 2, 6 and 9 respectively. For their proofs, we proceed as follows:

For the arbitrary fixed $R=1+\frac{1}{n}$, let us set $L^{*}:=y\left(L_{R}\right)$. According to Lemma 22 , the number $\rho_{1}:=1+c_{1}(R-1)$ can be chosen as $\bar{G}_{\rho_{1}}^{*} \subseteq$ G. Let $R_{1}:=1+\frac{\rho_{1}-1}{2}$. Analogously to the proof of Theorem 2, we define:

$$
\begin{gathered}
B_{n}^{*}(z):=\prod_{j=1}^{m} B_{j}^{*}(z)=\prod_{j=1}^{m} \frac{\Phi_{R}(z)-\Phi_{R}\left(z_{j}\right)}{1-\overline{\Phi_{R}\left(z_{j}\right)} \Phi_{R}(z)}, \\
\widetilde{F}_{n, p}^{*}(z):=\left(\frac{P_{n}(z)}{B_{m}^{*}(z) \Phi_{R}^{n+1}(z)}\right)^{\frac{p}{2}} .
\end{gathered}
$$

Then, by the Cauchy integral formula for a region $\Omega_{R_{1}}^{*}$, we have:

$$
\widetilde{F}_{n, p}^{*}(z)=-\frac{1}{2 \pi i} \int_{L_{R_{1}}^{*}} \widetilde{F}_{n, p}^{*}(\zeta) \frac{d \zeta}{\zeta-z}, z \in \Omega_{R_{1}}^{*} .
$$

By repeating the same reasoning as the proof of Theorem 2, we find:

$$
\left|P_{n}(z)\right| \preceq\left(\frac{\sqrt{n}}{d\left(z, L_{R_{1}}^{*}\right)}\right)^{\frac{2}{p}}\left\|P_{n}\right\|_{A_{p}(G)}|\Phi(z)|^{n+1}, z \in \Omega_{R_{1}}^{*} .
$$

Since

$$
d\left(z, L_{R_{1}}^{*}\right) \succeq d(z, L) \text { for } z \in \Omega,
$$

then, we have:

$$
\left|P_{n}(z)\right| \preceq\left(\frac{\sqrt{n}}{d(z, L)}\right)^{\frac{2}{p}}\left\|P_{n}\right\|_{A_{p}(G)}|\Phi(z)|^{n+1}, z \in \Omega .
$$

\subsection{Proof of Theorem 15}

Proof. In this case, for same $R, \rho_{1}, R_{1}$ and any $p \geq 1$ set:

$$
G_{n, p}(z):=\left(\frac{P_{n}(z)}{\Phi^{n+1}(z)}\right)^{p}, z \in \Omega^{*} .
$$

From the Cauchy integral formula, we have:

$$
G_{n, p}(z)=-\frac{1}{2 \pi i} \int_{L_{R_{1}}^{*}} G_{n, p}(\zeta) \frac{d \zeta}{\zeta-z}, z \in \Omega_{R_{1}}^{*}
$$

Then

$$
\left|G_{n, p}(z)\right| \preceq \frac{1}{d\left(z, L_{R_{1}}^{*}\right)} \int_{L_{R_{1}}^{*}}\left|P_{n}(\zeta)\right|^{p}|d \zeta|
$$


and, by repeating the same reasoning as the proof of Theorem 6 , we obtain:

$$
\left|P_{n}(z)\right| \preceq\left(\frac{n^{\frac{1}{\alpha}}}{d\left(z, L_{R_{1}}^{*}\right)}\right)^{\frac{1}{p}}\left\|P_{n}\right\|_{A_{p}(G)}\left|\Phi^{n+1}(z)\right|, z \in \Omega_{R_{1}}^{*}, p \geq 1 .
$$

According to (4.26), we complete the proof.

\subsection{Proof of Theorem 16}

Proof. Analogously to prove of the Theorem 9, according to the Cauchy integral representation, we have:

$$
G_{n, 1}(z)=-\frac{1}{2 \pi i} \int_{L_{R_{1}}^{*}} G_{n, 1}(\zeta) \frac{d \zeta}{\zeta-z}, z \in \Omega_{R_{1}}^{*}
$$

Then, by the proof of Theorem 9, we get:

$$
\begin{gathered}
\left|G_{n, 1}(z) \preceq \frac{1}{d\left(z, L_{R_{1}}^{*}\right)} \int_{L_{R_{1}}^{*}}\right| P_{n}(\zeta)|| d \zeta \mid \\
\leq \frac{1}{d\left(z, L_{R_{1}}^{*}\right)}\left(\int_{w \mid=R_{1}}\left|f_{n, p}(w)\right|^{p}|d w|\right)^{\frac{1}{p}} \cdot\left(\int_{|w|=R_{1}}\left|\Psi_{R}^{\prime}(w)\right|^{\left(1-\frac{2}{p}\right) q}|d w|\right)^{\frac{1}{q}} \\
\preceq \frac{1}{d\left(z, L_{R_{1}}^{*}\right)}\left(\int_{|w|=R_{1}}\left|f_{n, p}(w)\right|^{p}|d w|\right)^{\frac{1}{p}} \cdot\left(\int_{|w|=R_{1}}\left|\Psi_{R}^{\prime}(w)\right|^{2-q}|d w|\right)^{\frac{1}{q}}=: A_{n, p} \cdot B_{n, q},
\end{gathered}
$$

where $f_{n, p}(w):=P_{n}\left(\Psi_{R}(w)\right)\left(\Psi_{R}(w)\right)^{\frac{2}{p}}$ and $\frac{1}{p}+\frac{1}{q}=1$. From the Lemma 24, we have:

$$
A_{n, p} \preceq n^{\frac{1}{p}}\left\|P_{n}\right\|_{A_{p}(G)} .
$$

On the other hand, according to the estimation for $\left|\Psi_{R}^{\prime}\right|$ ( [10, Th.2.8]), we get:

$$
B_{n, q}^{q} \asymp \int_{|w|=R_{1}}\left(\frac{d\left(\Psi_{R}(w), L^{*}\right)}{|w|-1}\right)^{2-q}|d w| .
$$

Let $1<q<2$. Since $G \in Q_{\alpha}^{\beta}$, for $z^{*} \in L^{*}$ such that $d\left(\Psi_{R}(w), L^{*}\right)=$ $\left|\Psi_{R}(w)-z^{*}\right|$ and $w^{*}:=\Phi_{R}\left(z^{*}\right)$, we have:

$$
d\left(\Psi_{R}(w), L^{*}\right) \preceq\left|w-w^{*}\right|^{\beta} \preceq(|w|-1)^{\beta} .
$$


Then, from Lemma 20 and (4.28), we obtain:

$$
\begin{aligned}
B_{n, q}^{q} \preceq \int_{|w|=R_{1}}\left(\frac{(|w|-1)^{\beta}}{|w|-1}\right)^{2-q}|d w| \preceq \int_{|w|=R_{1}} \frac{|d w|}{(|w|-1)^{(1-\beta)(2-q)}} \\
\qquad \frac{1}{\left(R_{1}-1\right)^{(1-\beta)(2-q)} \preceq n^{(1-\beta)(2-q)} .}
\end{aligned}
$$

Let $q>2$. Let us put $d\left(\Psi_{R}(w), L^{*}\right):=\left|\widetilde{z}-z^{*}\right|, \widetilde{z} \in L_{R_{1}}^{*}$ and $\widetilde{w}:=\Phi_{R}(\widetilde{z})$, $w^{*}:=\Phi_{R}\left(z^{*}\right)$. According to (4.7), we get $\left|\widetilde{z}-z^{*}\right| \succeq\left|\widetilde{w}-w^{*}\right|^{\frac{1}{\alpha}}$. Then,

$$
\begin{aligned}
B_{n, q}^{q} & \asymp \int_{|w|=R_{1}}\left(\frac{|w|-1}{d\left(\Psi_{R}(w), L^{*}\right)}\right)^{q-2}|d w| \\
& \preceq \int_{|w|=R_{1}}\left(\frac{|w|-1}{\left|\widetilde{w}-w^{*}\right|^{\frac{1}{\alpha}}}\right)^{q-2}|d w| \preceq \int_{|w|=R_{1}} \frac{|d w|}{\left|\widetilde{w}-w^{*}\right|^{\left(\frac{1}{\alpha}-1\right)(q-2)}} \\
& \preceq n^{\left(\frac{1}{\alpha}-1\right)(q-2)-1}, \quad\left(\frac{1}{\alpha}-1\right)(q-2)<1 .
\end{aligned}
$$

By combining (4.27)-(4.30), we obtain:

$$
\left|P_{n}(z)\right| \preceq \frac{|\Phi(z)|^{n+1}}{d\left(z, L_{R_{1}}^{*}\right)}\left\|P_{n}\right\|_{A_{p}(G)}\left\{\begin{array}{cc}
n^{\frac{1}{\alpha}\left(\frac{2}{p}-1\right)}, & 2-\alpha<p<2 \\
\sqrt{n}, & p=2, \\
n^{(1-\beta)\left(1-\frac{2}{p}\right)} & \beta>0, \quad p>2,
\end{array} \quad z \in \Omega_{R_{1}}^{*} .\right.
$$

According to (4.26), we complete the proof.

\subsection{Proof of Theorem 17.}

Proof. a) Let $Q_{n}^{*}(z):=\sum_{j=0}^{n}(j+1) z^{j}, G_{1}^{*}=B$ and $p=2$. In this case,

$$
\left\|Q_{n}^{*}\right\|_{C(\bar{G})}=\frac{(n+1)(n+2)}{2} ;\left\|Q_{n}^{*}\right\|_{A_{2}(G)}=\sqrt{\frac{\pi(n+1)(n+2)}{2}} .
$$

Then, we have

$$
\left\|Q_{n}^{*}\right\|_{C(\bar{G})} \geq \frac{1}{\sqrt{2 \pi}} n\left\|Q_{n}^{*}\right\|_{A_{2}(G)} .
$$

b) Let the region $G_{2}^{*} \subset \mathbb{C}$ bounded by smooth curve $L=\partial G \in C_{\theta}$. According to the "three-point" criterion [17, p.100] the curve $L$ is quasiconformal. Denote by $\left\{K_{n}(z)\right\}$, $\operatorname{deg} K_{n}=n, n=0,1,2, \ldots$, the system of Bergman polynomials for region $G$, i.e. $K_{n}(z):=\alpha_{n} z^{n}+\alpha_{n-1} z^{n-1}+\ldots+\alpha_{0}, \alpha_{n}>0$ and

$$
\iint_{G} K_{n}(z) \overline{K_{m}(z)} d \sigma_{z}=\delta_{n, m}
$$


where $\delta_{n, m}$ is the symbol Kronecker. Let $\overline{G_{2}^{*}}$ be a closure of convex hull of $G_{2}^{*}$ and $F:=C \overline{G_{2}^{*}}$. It is well known [14, p.245], that zeros of Bergman polynomials $K_{n}(z)$ are contained in $\overline{G_{2}^{*}}$. According to [2], for arbitrary quasidisks we have:

$$
K_{n}(z)=\alpha_{n} \rho^{n+1} \Phi^{n}(z) \Phi^{\prime}(z) A_{n}(z), z \in F \Subset \Omega,
$$

where

$$
\sqrt{\frac{n+1}{\pi}} \leq \alpha_{n} \rho^{n+1} \leq c_{1} \sqrt{\frac{n+1}{\pi}}
$$

for some $c_{1}=c_{1}(G)>1$ and

$$
c_{2} \leq\left|A_{n}(z)\right| \leq 1+\frac{c_{3}}{\sqrt{|\Phi(z)|-1}}
$$

for some $c_{i}=c_{i}(G)>0, i=2,3$. Therefore, since $\left\|K_{n}\right\|_{A_{2}(G)}=1$, we have

$$
\begin{aligned}
\left|K_{n}(z)\right| & \geq c_{2} \sqrt{\frac{n+1}{\pi}}|\Phi(z)|^{n} \frac{|\Phi(z)|-1}{d(z, L)} \\
& \geq c_{3} \frac{\sqrt{n}}{d(z, L)}|\Phi(z)|^{n+1}\left(1-\frac{1}{|\Phi(z)|}\right) \\
& \geq c_{4} \frac{\sqrt{n}}{d(z, L)}|\Phi(z)|^{n+1}\left\|K_{n}\right\|_{A_{2}(G)} .
\end{aligned}
$$

\section{References}

[1] Abdullayev F.G. \& Andrievskii V.V., On the orthogonal polynomials in the domains with K -quasiconformal boundary. Izv. Akad. Nauk Azerb. SSR., Ser. FTM, 1983, No:1, pp. 3-7.

[2] Abdullayev F.G., Dissertation (Ph.D.), Donetsk, 1986, 120 p.

[3] Abdullayev F.G., On the some properties of the orthogonal polynomials over the region of the complex plane (Part III), Ukr.Math.J., 2001, Vol.53, No:12, pp.19341948.

[4] Abdullayev F.G., The properties of the orthogonal polynomials with weight having singulerity on the boundary contour, J. of Comp. Anal. and Appl., 2004, Vol. 6, No: 1, pp. 43-59.

[5] Abdullayev F.G., Deger U., On the orthogonal polynomials with weight having singularity on the boundary of regions of the complex plane, Bull. Belg. Math. Soc., 2009, Vol 16, No:2, pp.235-250.

[6] Abdullayev F. G., Gün C.D., On the behavior of the algebraic polynomials in regions with piecewise smooth boundary without cusps, Ann.Polon.Math., 2014, 111, pp.39-58. 
[7] Abdullayev, F. G., Özkartepe N.P., On the Behavior of the Algebraic Polynomial in Unbounded Reg1ons with Piecewise Dini-Smooth Boundary, Ukranian Mathematical Journal, 2014, 66 (5), pp.579-597.

[8] Ahlfors L, Lectures on Quasiconformal Mappings. Princeton, NJ: Van Nostrand, 1966.

[9] Andrievskii V.V., Constructive characterization of the harmonic functions in domains with quasiconformal boundary, In: Quasiconformal continuation and Approximation by function in the set of the complex plane. Kiev, 1985. [in Russian]

[10] Andrievskii V.V., Belyi V.I. \& Dzyadyk V.K., Conformal invariants in constructive theory of functions of complex plane. Atlanta:World Federation Publ.Com., 1995.

[11] Andrievskii V. V., Weighted Polynomial Inequalities in the Complex Plan, Journal of Approximation Theory, 2012, 164 (9), 1165-1183.

[12] Belinskii P.P., General Properties of Quasiconformal Mappings, Nauka, Sib. otd., Novosibirsk, 1974. [in Russian]

[13] Bernstein S.N., Sur l'ordre de la meilleure approximation des fonctions continues par les polynomes de degre donne, Mem. Cl.Sci. Acad.Roy. Belgique, 1912, Vol.4 No:2, 1-103.

[14] Davis P.J., Interpolation and Approximation. Blaisdell Publishing Company, 1963.

[15] Faber. G., Über nach Polynomen fortschreitende Reihen, Sitzungsberichte der Bayrischen Akademie der Wissenschaften, 1922, pp.157-178.

[16] Hille E., Szegö G., Tamarkin J.D., On some generalization of a theorem of A.Markoff , Duke Math., 1937, 3, p. 729-739.

[17] Lehto O., Virtanen K.I., Quasiconformal Mapping in the Plane, Springer Verlag, Berlin, 1973.

[18] Lesley F.D., Hölder continuity of conformal mappings at the boundary via the strip method, Indiana Univ. Math. J., 1982, 31, 341-354.

[19] Mamedhanov D.I., On Nikolskii-type inequalities with new characteristics, Doklady Mathematics, 2010, 82, 882-883.

[20] Milovanovic G. V., Mitrinovic D.S., and Rassias Th.M., Topics in Polynomials: Extremal Problems, Inequalities, Zeros, Singapore, World Scientific, 1994.

[21] Nikol'skii S. M., Approximation of Function of Several Variable and Imbeding Theorems, New-York, Springer-Verlag, 1975. 
[22] Pommerenke Ch., Univalent Functions, Göttingen, Vandenhoeck \& Ruprecht, 1975.

[23] Rickman S., Characterisation of quasiconformal arcs, Ann. Acad. Sci. Fenn., Ser. A, Mathematica., 1966, 395,30 p.

[24] Stylianopoulos N., Fine asymptotics for Bergman orthogonal polynomials over domains with corners, CMFT 2009, Ankara, June 2009.

[25] Stylianopoulos N., Strong asymptotics for Bergman polynomials over domains with corners and applications, Const. Approx., 33, 2013, 59-100.

[26] Warschawski S.E., On differentiability at the boundary in conformal mapping, Proc. Amer. Math. Soc.,12, 1961, 614-620.

[27] Warschawski S.E., On Hölder continuity at the boundary in conformal maps, L. of Math. and Mech. 1968, 18, 423-427.

[28] Walsh J.L., Interpolation and Approximation by Rational Functions in the Complex Domain, AMS, 1960.

Mersin University, Faculty of Arts and Science, Department of Mathematics, 33343 Mersin-TURKEY.

emails:fabdul@mersin.edu.tr; fahreddinabdullayev@gmail.com; pelinozkartepe@gmail.com 